\title{
Novel Handheld Device to Measure the Nasal Expiratory Flow Rate of the Two Nasal Cavities Individually and Simultaneously
}

\author{
Thimira S. Thilakarathna, Mahesh Edirisinghe* \\ Department of Physics, University of Colombo, Colombo 03, 00300, Sri Lanka
}

Corresponding Author Email: mahesh@phys.cmb.ac.lk

https://doi.org/10.18280/i2m.200102

Received: 18 November 2020

Accepted: 23 January 2021

\section{Keywords:}

medical device, nasal airflow detection, exhaled airflow rate, breathing style, embedded system

\begin{abstract}
This paper reveals a novel handheld device to measure the peak nasal expiratory flow rate of the two nasal cavities individually and simultaneously. Significantly, when using this device, the patient needs to inhale from the mouth and exhale from the nose, as a usual breath, and importantly there is no need to take extra effort to exhale. The key detection mechanism used is the anemometer technique and the measurement is done by using an optical wheel encoder and an ATmega328p microcontroller. The device is capable of measuring the exhaled airflow rate with an accuracy of \pm 1 LPM. It also allows measuring the number of breaths per minute together with the respective temperature and the relative humidity of the exhaled airflow of the two nasal cavities separately. Two DHT22 sensor modules are used to measure relative humidity and temperature with an accuracy of $\pm 1 \% \mathrm{RH}$ and $\pm 0.5^{\circ} \mathrm{C}$ respectively. This can be used by a Rhinologist as an initial diagnosing tool for several diseases, as the device allows them to get an idea about the patient's exhaling behavior of the nasal airflow instantly. The device would be beneficial in the sports sector as a physical fitness indicator by means of the breathing style.
\end{abstract}

\section{INTRODUCTION}

In human health, as stated in previous studies [1-3], the respiratory system is very important as it contains a series of organs responsible for transporting air into the lungs, filtering oxygen to the red blood cells, and exhaling carbon dioxide. As stated by researches $[2,3]$, the nose is an important organ in the respiratory system. It is the organ that inhales while filtering out dust, germs, and irritants of air and exhales the air out of the respiratory system [2]. On the other hand, as highlighted in previous studies [1-4], exhaled airflow rate and breathing style will provide some indications on different diseases and respective symptoms.

As stated in previous studies [1-3], there could be more syndromes other than swelling of erectile tissue such as the deviated septum, nasal polyps, rhinitis, etc... which influence the change in breathing style. On the other hand, it is a wellknown general fact that the nasal airflow could take as an initial diagnosing method for some diseases. In nasal airflow, the nasal cycle is an important component and it is expected that both left and right nasal exhaled airflow rates should be the same for a healthy person $[3,5]$.

Examining the expiratory airflow can be done by exploring the historical information of the patient together with a physical checkup [3]. Then the objective measurements can be taken from the expiratory airflow on the mouth or nose in several ways [6]. However, as an initial diagnosis mechanism, most of the time, doctors use their fingers to detect and get an idea about the airflow of the nasal expiratory air. On the other hand, there are some tools and equipment that have been developed to be used at hospitals, to assess the patient's exhaled airflow.

In the early stage, there were two historical techniques called "hygrometry test" and "hum test" as an initial diagnosis mechanism [3, 7]. In "hygrometry test" it measures the fog diameter appear on a mirror due to the patient's breath and in "hum test", it tests the quality of the sound created due to the humming sound generated by the patient through one nasal airway [3, 7]. However, in both cases, the patient dissatisfaction rate was very high $[3,7]$.

In a modern medical laboratory environment, a CT scan is used to assess expiratory airflow but could cause exposure to radiation [8]. Peak Nasal Inspiratory Flow (PNIF) is another method that measures the nasal airflow in liters per minute (LPM) $[8,9]$. In this test, a PNIF meter with the mask that covers the nose and mouth together can be coupled to a computer system and the measurements can be recorded as well $[8,9]$. However, the measurements from the PNIF meter depends on the effort of the patient under the guidance of the physician [8]. In Acoustic Rhinometry (AR) test, it measures the cross-sectional area of the nose with respect to the distance from the nostrils $[3,8,10]$. The principle of this device is to analyze the sound wave reflections from the nose after undergoing a 10 to 20 minutes relaxing time $[3,8,10]$. However, before taking the real measurements, the equipment has to be calibrated while keeping the head of the patient steady [3, 8]. Nevertheless, AR requires minimal patient cooperation than the PNIF test $[3,8]$.

Rhinomanometry (RM) test is another objective measurement of nasal airflow which converts the transnasal pressure into an electrical signal using a pressure transducer which relates the respective airflow $[3,8]$. In this test, the patient should sit and rest for 20 minutes and should breathe spontaneously while taking the measurement $[3,8]$.

Peak expiratory flow rate measurement (PEFR test) is another test to measure the flow of air coming in and out of the 
lungs and it is mostly carried out for patients who have asthma [8]. This technique is done by using the oral expiratory flow but not the nasal flow which measures a person's maximum speed of expiration and gives the volume that flow in liters per minute [8]. However, breath out should be done by the patient with the fastest possible speed [8].

As indicated in previous studies [8, 11], there were different scientific principles used as the transducer for measuring nasal exhaled airflow rate. As stated in previous studies [11, 12], pneumotachometer or respiratory plethysmograph is the most commonly used system for such measurements. However, a previous study [12] had proposed an alternative technique that measures the exhaled air flow rate using a pressure sensor but it can be used only to detect airflow rate from the mouth with the sealed nose.

Reviewing the aforementioned existing mechanisms and available instrumentation for measuring or examining the nasal exhaled airflow, it was noted that a significant effort from the patient is needed to get the correct diagnosis [8]. A major drawback of such commercially available measuring devices is that as of the present techniques, it is not possible to detect the nasal airflow rate of the two nasal cavities individually and simultaneously [8]. On the other hand, if it is a portable device with less involvement by the patient, it could be a useful device as an initial diagnosing method. Henceforth, if such a portable device is available on the physicians' table, somewhat similar to a sphygmomanometer and a stethoscope, it will be a vital tool for efficient and comfortable diagnosis. As emphasized and highlighted in a previous study [2], such a device can be used as an initial diagnostic tool for Septum Deviation, Nasal Polyps, Nosebleeds, Rhinitis, Nasal Fractions, Nasal Cycles and Breathing Style, etc... It is important to mention that, in the USA, about 31 million or more patients are reported every year with nasal obstruction due to different diseases [13]. A routine evaluation of nasal expiratory peak flow rate of elderly people is also important for many diagnostics [13]. Moreover, knowing the nasal expiratory flow rate with the respective temperature and humidity level is essential as it reflects the healthiness of the respiratory system of a human $[14,15]$.

The main objective of this study is to develop a portable handheld nasal exhaled air flow rate measuring device with an attempt to address the aforementioned issues with many specific additional features. This paper reveals a novel nasal flow measuring device as an outcome of the study named; Nasal Airflow Detection and Measuring System (NAMS) [16] In this paper, a device for measuring nasal exhaled peak airflow rate from both right and left nostrils separately and simultaneously is introduced, which is the unique novelty of the device [16]. The mechanism introduced to measure the number of breaths per minute (to monitor the breathing style) of a patient together with the temperature and the relative humidity of the exhaled airflow of the two nasal cavities separately is also discussed. On the other hand, when developing medical devices, it is important to report the applicable symptoms and contraindications of such devices [17]. Therefore, an attempt has been made to recognize the applicable symptoms and contraindications of the developed device. Furthermore, identifying suitable disinfecting mechanisms from the available procedures are also addressed in this paper [18-22].

\section{INNOVATIVE METHODOLOGY}

The foremost innovative capability of the developed device is that it can measure nasal airflow rate separately and simultaneously in the two nasal cavities. It follows a novel methodology to detect and measure nasal airflow rate by, passing the nasal airflow through a propeller (rotatable vein) without applying positive or negative pressure using an external pressure source. The developed device comprises of two separate tubes that connect the two nostrils so that it allows two independent airflow paths for the two nasal cavities. Moreover, it consists of two separate compartments such that they have two propellers that only rotate when air flows through the said tubes. Furthermore, each propeller is coupled to a speed measuring device which comprises of two identical wheel encoding sensors. So that the device is capable of calculating nasal airflow rate separately and simultaneously using the said wheel encoding sensor. The device also comprising a temperature sensor and a humidity sensor in each of the aforementioned two separate tubes which connects the two nostrils intending to detect temperature and relative humidity of the nasal exhaled airflow of two nasal cavities separately and simultaneously. A microcontroller-based data acquisition and processing system has also been incorporated together with the data storing and retrieving facility into the developed device to process the data from the sensors and to store processed or sensor-data for future references. Furthermore, the device comprises an OLED (Organic LightEmitting Diodes) type display to indicate sensor-data and other relevant information during the operative stage. As the expectation was to develop it as a handheld device, a rechargeable battery pack has also been coupled and a hardware interface has been developed to download the stored data into a PC for further analysis of the patients' progress.

\section{DESIGN AND CONSTRUCTION}

The design and construction of the developed device comprises of three main sections namely; Mechanical Parts, Electronic Parts \& Accessories, and Device Validation Process.

\subsection{Design of mechanical parts}

Most of the mechanical parts are custom-designed and 3D printed. The designing of those parts were done by using the SOLIDWORKS 2016 software. As the material for 3D printing, PLA (Poly-Lactic Acid) polymer was used. In this novel device, the air from the nasal cavities had to direct through the sensing system to measure the flow rate. Henceforth, an innovative approach had been made to design the main body of the device together with side covers, front cover, back cover, and two propellers (sensing fans). 
(a)

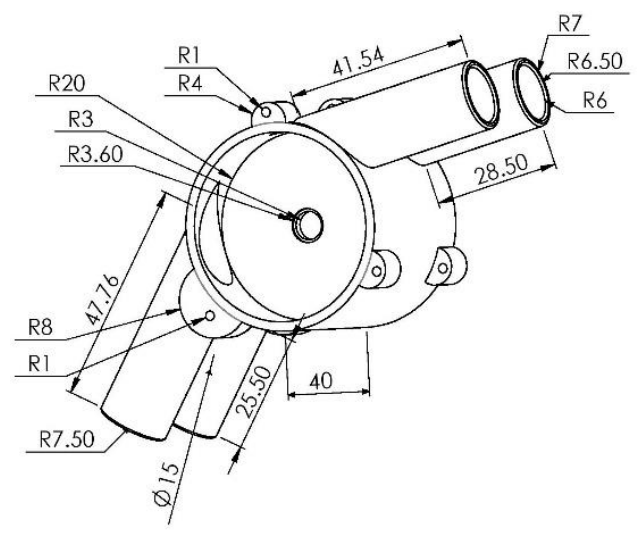

All dimensions are in millimeters $(\mathrm{mm})$. (b)

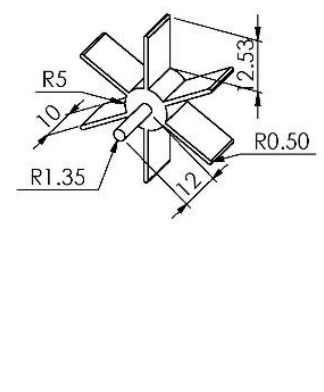

Figure 1. Main parts of the device (a) tubes to connect nostrils (b) sensing fan (c) final assembly (c)

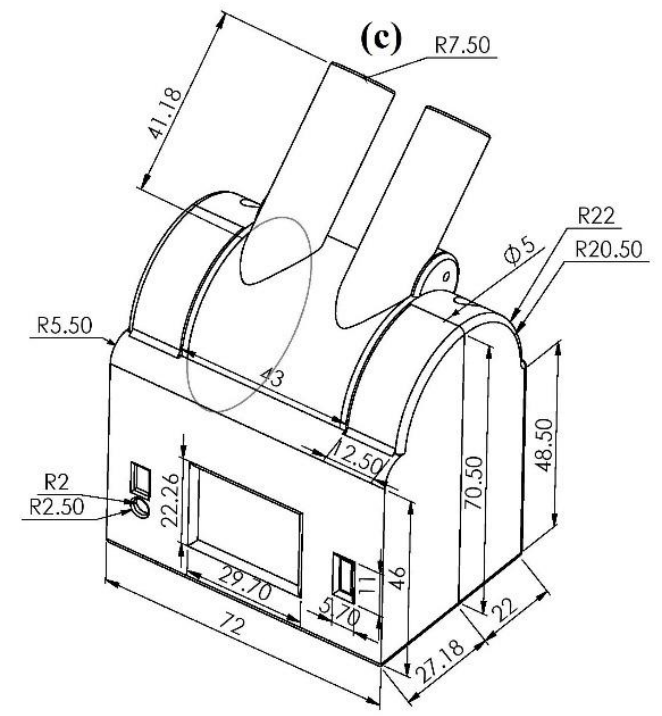

the aforementioned rectangular shape to mount the data communication interface and charging port and for necessary switches. Both front and back cover protects the moving parts, electronic parts, and accessories. Figure 1(c) shows the final assembly of the design with the specific dimensions of the respective parts.

\subsection{Design of electronic parts and accessories}

Figure 2 shows the block diagram of the electronic system of NAMS. As illustrated in Figure 2, the electronic system of the device consists of its central processing unit which comprises of microcontroller-based data acquisition and processing unit with its required accessories, together with the display unit, power supply unit, and charging and hardware interface unit.

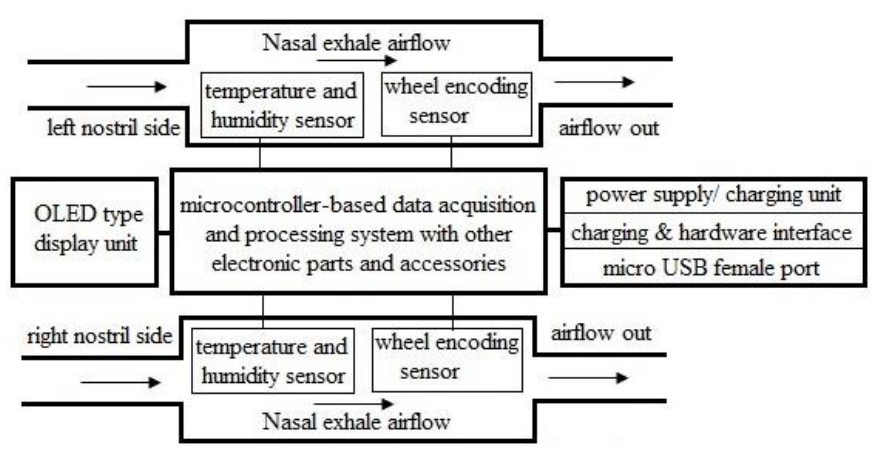

Figure 2. Block diagram of the electronic system of NAMS

To process the sensor-data and output information, a microcontroller unit that comes with the ATmega328p microcontroller was used. Since the Arduino bootloader was installed on the microcontroller unit, it allows to program using the Arduino IDE.

To detect the speed of the two sensing fans, two photoelectric speed sensors with a rotating encoder disk for each are used. The encoder disc which has 100 holes is fitted to the shaft of the fan. So from a sensor, it counts 100 steps for a unit revolution. The device displays the number of 10 steps for $50 \mathrm{~ms}$, which is the sensitivity of the device. In order to measure both relative humidity and temperature, the DHT22 
sensor module is used. This sensor uses the 1-wire protocol to communicate with the microcontroller. The sensitivity of the relative humidity and temperature sensors available in DHT22 are $0.1 \%$ and $0.1^{\circ} \mathrm{C}$ respectively whereas the accuracy of the two sensors are $\pm \%$ and $\pm 0.5^{\circ} \mathrm{C}$ respectively. There are two DHT22 sensor modules used for both left and right sides.

As the power source, a $550 \mathrm{mAh}, 3.7 \mathrm{~V} \mathrm{Li}$-ion rechargeable battery pack is used. To charge the Li-ion battery and to get 5 $\mathrm{V}$ output for the functionality of the electronics in the device, a USB charge/ DC-DC boost converter module of a lithium battery power bank is used. It contains a micro USB female port for charging and a hardware interface has also been developed to couple the device into a PC so that downloading the stored data and carry out further analysis of the patients' progress is also possible. An OLED display module is used to display sensor-data and other relevant information during both the operative and review modes. This display module connects the microcontroller unit with the $\mathrm{I}^{2} \mathrm{C}$ bus which has a display resolution of $128 \times 64$.

\subsection{Device validation process}

The validation process of the developed device was done in two main phases. As the initial validation process, validation of partially assembled electronics was done. Before assembling the electronic components, each part had been individually tested and re-tested again after partial assembling. At this stage, all the required adjustments and fine-tuning were made as per the test results.

Figure 3 shows the fully assembled novel device which was developed as a Nasal Airflow Detection and Measuring System (NAMS) after assembling all the components, mechanical parts, accessories, and all 3D printed side covers.

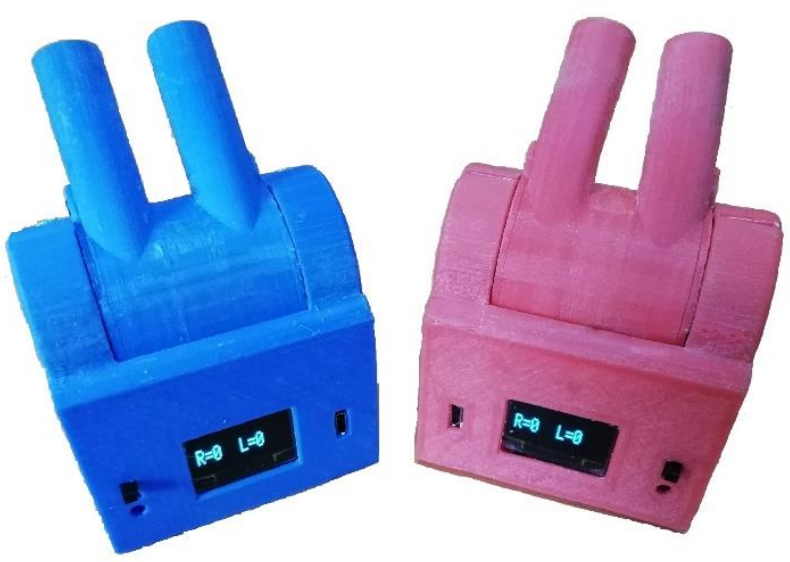

Figure 3. Fully assembled device (NAMS)

As the final phase of the validation process, another test was done to reveal the stability of the fully assembled system. In this test, the output of the device as flow rate counts for both left and right airflow were recorded for different flow rates in every time interval of 120 seconds up to six readings for each flow rate. Flow rates were varied between 5 LPM and 40 LPM with 5 LPM increments. Table 1 shows the tabulated observations under the aforesaid test. It is important to mention that, flow rate counts for both left and right tubes were noted zero when there was no flow rate applied.

According to Table 1, flow rate counts given by the device, for all the measured flow rates under observed time intervals are with adequate consistency within an accuracy of \pm 1 count. Moreover, the developed NAMS is not calibrated to measure the absolute value of the flow rate but only to take the relative measure. However, as per the stability test results, to get a relationship between the absolute flow rate and flow rate counts given by the device, data indicated in Table 2 that has been extracted from Table 1 was used. As the left side and right side values are similar to a given flow rate, both left and right readings can be considered as the same value. Furthermore, an airflow with different flow rates as mentioned above has been applied to only one tube while covering the other tube and vice versa. During this stage, it was noted that a zero count for the closed tube whereas the other tube followed the variation as stated in Table 1 . Moreover, it is important to mention that during the test conducted to generate Table 1, the airflow has been fed to both the left and right tubes simultaneously as a parallel test. Henceforth, it could confirm that the strength and airtightness of the device met the requirements of long-term recycling use. However, regular calibration is recommended for long-term usage.

Figure 4 illustrates the graphical relationship of absolute flow rate and flow rate counts generated as per the information given in Table 2. As indicated in Figure 4, a linear relationship between the absolute flow rate and flow rate counts has been observed with a gradient of 0.6535 and $\mathrm{R}^{2}$ value of 0.9981 . Therefore, with the use of this relationship, one can estimate the absolute flow rate of the nasal exhaled airflow of the particular person.



Figure 4. Graphical relationship of absolute flow rate and flow rate counts

Table 1. Right and left flow rate counts for different flow rates at the stability test

\begin{tabular}{|c|c|c|c|c|c|c|c|c|c|c|c|c|c|c|c|c|}
\hline \multirow{3}{*}{$\begin{array}{c}\text { Time } \\
\text { (sec) } \\
\pm 1\end{array}$} & \multicolumn{16}{|c|}{ Left $(L)$ and Right $(R)$ flow rate counts $( \pm 1)$ with different flow rates in LPM $( \pm 1)$} \\
\hline & \multicolumn{2}{|c|}{5 LPM } & \multicolumn{2}{|c|}{10 LPM } & \multicolumn{2}{|c|}{15 LPM } & \multicolumn{2}{|c|}{20 LPM } & \multicolumn{2}{|c|}{25 LPM } & \multicolumn{2}{|c|}{ 30 LPM } & \multicolumn{2}{|c|}{35 LPM } & \multicolumn{2}{|c|}{40 LPM } \\
\hline & $\mathbf{L}$ & $\mathbf{R}$ & $\mathbf{L}$ & $\mathbf{R}$ & $\mathbf{L}$ & $\mathbf{R}$ & $\mathbf{L}$ & $\mathbf{R}$ & $\mathbf{L}$ & $\mathbf{R}$ & $\mathbf{L}$ & $\mathbf{R}$ & $\mathbf{L}$ & $\mathbf{R}$ & $\mathbf{L}$ & $\mathbf{R}$ \\
\hline $\mathbf{0}$ & 6 & 6 & 14 & 14 & 21 & 22 & 32 & 32 & 38 & 38 & 46 & 46 & 54 & 54 & 61 & 61 \\
\hline 120 & 6 & 6 & 14 & 14 & 22 & 22 & 30 & 30 & 38 & 38 & 46 & 46 & 53 & 54 & 61 & 62 \\
\hline 240 & 5 & 7 & 14 & 14 & 22 & 22 & 30 & 30 & 38 & 37 & 46 & 46 & 54 & 54 & 62 & 62 \\
\hline 360 & 6 & 6 & 14 & 14 & 22 & 22 & 30 & 30 & 38 & 38 & 46 & 46 & 54 & 54 & 62 & 62 \\
\hline 480 & 6 & 6 & 14 & 14 & 22 & 22 & 30 & 30 & 38 & 38 & 46 & 46 & 54 & 54 & 62 & 62 \\
\hline 600 & 6 & 6 & 14 & 14 & 21 & 22 & 30 & 30 & 38 & 38 & 46 & 46 & 54 & 54 & 62 & 62 \\
\hline
\end{tabular}


Table 2. Mode values of the right and left readings for different flow rates

\begin{tabular}{clllllllll}
\hline Flow rate counts $( \pm \mathbf{1})$ & 0 & 6 & 14 & 22 & 30 & 38 & 46 & 54 & 62 \\
Absolute Flow rate in LPM $( \pm \mathbf{1})$ & 0 & 5 & 10 & 15 & 20 & 25 & 30 & 35 & 40 \\
\hline
\end{tabular}

\section{OPERATIONAL PROCEDURE}

The developed device functions under two specific modes namely; Patient Selection Mode and Measurement Mode. When the device is switched on, by default it is on the Patient selection mode, and the OLED screen displays the initial patient number as zero. By replication of pressing the device push button for less than $500 \mathrm{~ms}$ (short-press) as required, the patient number can be changed. After selecting the respective patient number by keep pressing the device push button for more than $500 \mathrm{~ms}$ (long-press), the device enters the Measurement mode.

In this mode, the device is ready to take the measurements, and the display shows the instantaneous flow rate count while taking other measurements at the same time. After taking the measurements of a patient, the data can be saved with a longpress. After saving the measured data, a cycle of shot-press allows the user to review the same which includes peak airflow rate count for both left and right nasal exhaled airflow, breathing rate (number of breaths per minute), temperature, and humidity. The device is currently capable of storing up to ten patients' data at a time using the internal storage of the microcontroller unit. Moreover, these data can be downloaded to a computer by using the special application software that developed specifically for this. Figure 5 shows the interface of the application software.

To download the patient data to a computer, the device should be connected to the computer using a USB cable. When the device is connected to a computer, Retrieve Data From Device button should be clicked to download all stored data from the device. This application software allows the user to retrieve the measured data of each patient, and the extracted data can be saved as individual files with the patient name. This application software also allows clearing all the measured data from the device. The communication between the computer and the device occurs as a UART communication. According to the sample test done, the retrieved and tabulated data from the device for five individuals are given in Table 3 as a performance of the device.

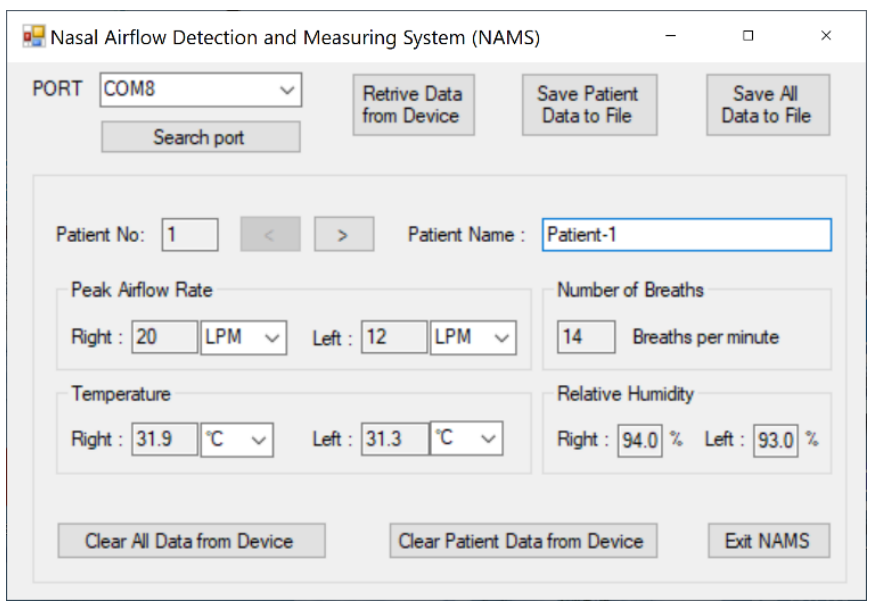

Figure 5. NAMS interface of the application software

Table 3. Retrieved sample data from the device with a $0.1 \%$ and $0.1^{\circ} \mathrm{C}$ resolution for relative humidity and temperature

\begin{tabular}{ccccccc}
\hline Patient No & & $\mathbf{1}$ & $\mathbf{2}$ & $\mathbf{3}$ & $\mathbf{4}$ & $\mathbf{5}$ \\
\hline Patient Name & & Patient-1 & Patient-2 & Patient-3 & Patient-4 & Patient-5 \\
\hline \multirow{2}{*}{ Peak Airflow Rate (LPM) } & Right & $20 \pm 1$ & $11 \pm 1$ & $18 \pm 1$ & $22 \pm 1$ & $23 \pm 1$ \\
\cline { 2 - 7 } & Left & $12 \pm 1$ & $15 \pm 1$ & $19 \pm 1$ & $24 \pm 1$ & $26 \pm 1$ \\
\hline No. of Breaths/ minute & $14 \pm 1$ & $9 \pm 1$ & $12 \pm 1$ & $10 \pm 1$ & $9 \pm 1$ \\
\hline \multirow{2}{*}{ Temperature $\left({ }^{\circ} \mathbf{C}\right)$} & Right & $31.9 \pm 0.5$ & $31.8 \pm 0.5$ & $31.4 \pm 0.5$ & $31.1 \pm 0.5$ & $31.6 \pm 0.5$ \\
\cline { 2 - 7 } & Left & $31.3 \pm 0.5$ & $32.5 \pm 0.5$ & $31.9 \pm 0.5$ & $32.0 \pm 0.5$ & $32.1 \pm 0.5$ \\
\hline \multirow{2}{*}{ Relative Humidity (\%) } & Right & $94.0 \pm 1$ & $95.0 \pm 1$ & $91.0 \pm 1$ & $95.0 \pm 1$ & $94.0 \pm 1$ \\
\cline { 2 - 7 } & Left & $93.0 \pm 1$ & $95.0 \pm 1$ & $92.0 \pm 1$ & $95.0 \pm 1$ & $94.0 \pm 1$ \\
\hline
\end{tabular}

\section{DISCUSSION}

The developed device namely Nasal Airflow Detection and Measuring System (NAMS) is specially designed to measure the exhaled flow rate of the two nasal cavities individually and simultaneously. Importantly, when using this device, the patient needs to inhale from the mouth and exhaled from the nose, as a usual breath, and generally no need to take extra effort to exhale. This device is developed as a handheld device so that it can be used as an initial investigative tool to observe a patient's exhaled airflow behavior of the nose. Authors suggest that NAMS would be a very useful handheld device as an initial diagnosing method for several diseases such as Septum Deviation, Nasal Polyps, Nosebleeds, Rhinitis, Nasal Fractions, Nasal Cycles and Breathing Style, etc... as suggested in previous studies [1-3].

The device, at the initial design stage, indicates the airflow rate not as an absolute measurement of the airflow but as flow rate counts. However, by referring to the transfer function as stated in Eq. (1), generated for the absolute flow rate counts in terms of the flow rate counts as exemplified in Figure 4 (the linear relationship between the absolute flow rate and flow rate counts), it is possible to calculate the airflow rate in LPM. 
Henceforth, the application software has been developed as indicated in Figure 5, which provides the nasal exhaled airflow rate as an absolute value with an accuracy of \pm 1 LPM, by multiplying the observed flow rate counts by the gradient (0.6535) of the Eq. (1). Even though measuring the exhaled flow rate of the two nasal cavities individually and simultaneously was the prime objective in this work, few value additions were also introduced as additional features. As such, the developed device is also capable of measuring the temperature and the relative humidity of the exhaled airflow of the two nasal cavities in parallel to the airflow rate measurements. Another unique feature added was monitoring the breathing style by measuring the number of breaths per minute and henceforth this device would be beneficial in the sports sector as a physical fitness indicator. The breathing rate is calculated by dividing the total number of local peaks of the instantaneous exhale flow rate by the total span time as shown in Eq. (2). The Total Span Time is the time between the first peak and the last peak detected by the device.

$$
\text { Breathing Rate }=\frac{\text { Number of Peaks Detected }}{\text { Total Span Time }}
$$

The developed device is capable of detecting the nasal expiratory flow rate of the two nasal cavities individually and simultaneously with an accuracy of \pm 1 LPM, whereas the commonly available PNIF meters and PEFR meters are detecting the overall nasal exhaled flow rates with a typical accuracy of \pm 5 LPM or above $[8,11]$. While using the device, as shown in Figure 6, it should be held by the patient and the nosepieces should be inserted into the nostrils. Then the patient has to exhale through the nose as normal breathing. As the display which shows the reading, is located at the front of the device, the physician will be able to see the values that the device shows. This allows a Rhinologist to get an idea about a patient's exhaling behavior of the nasal airflow. So the nasal resistance, deformations and other airflow related diseases can be instantly identified.

The developed device does not contain a separate nosepiece as this is a prototype. Nevertheless, when manufacturing on large scale and using on patients, it is better to use separate disposable nosepiece when attaching the device to the nose. So the geometry of the device can be modified to that level by shortening the current nosepiece and attaching disposable nosepieces. If nosepieces are in disposable form, it allows manufacturing nosepieces in different sizes. So this will allow using the device on any kind of age group as the current prototype has only a fixed size nosepiece.

While using the device, air leakage can occur between the nostrils and the nosepieces without passing through the device. This would result to get incorrect readings. A possible solution for this is to apply a viscous gel to prevent the leakage between the nostril and the nosepiece. After using it on a patient, the disposable nosepiece can be removed from the device and if there is excess gel on the device, it should be removed.

When discussing about the material that was used to 3Dprint the device, PLA was used which is a more common material in 3D printing. Importantly, this material is not toxic at solid-state. The microcontroller unit used in the device is Arduino pro mini. It contains an ATmega328p microcontroller, which comes from the AVR family manufactured by ATMEL Corporation. The Arduino platform, which supports the unit

for programming and the interrupt handling capability of the microcontroller is very special. Those facts were considered when choosing a microcontroller. Also, small in size and lower power consumption are other advantages of this microcontroller unit. As this device uses its on-board power, it becomes a portable device. Once it is charged for an hour, it can be continuously used for about four hours.

When handling the device, physical shocks to the device should be avoided. If the device experiences any physical shock, recalibration is recommended. Moreover, the glass transition temperature of the PLA is above $60^{\circ} \mathrm{C}$, with a $\pm 5^{\circ} \mathrm{C}$ safe margin [23, 24]. Furthermore, the manufacturer recommended upper limits of the temperature values under both operating and storing for electronics parts used in the device are also above $55^{\circ} \mathrm{C}$. Therefore, it is recommended that the device should not expose to temperatures above $55^{\circ} \mathrm{C}$.

Even though it is a mandatory requirement to flush and disinfect the device regularly and after taking measurements, applying any liquid is not recommended because it could affect the electronics and the performance of the moving mechanical parts of the device [18]. Low-Temperature Hydrogen Peroxide gas plasma or UV disinfection techniques are recommended for disinfecting the device [19-22].

There could be few contraindications applied to the device. Patients who are having lower awareness of handling the device properly or inability to look after their airway or if they are not cooperative are important to recognize and should not use the device for measurements. The device is not recommended for use for patients who are with anxiety, trauma, cardiorespiratory status, respiratory arrest, facial/ nasal injuries, copious respiratory secretions, nausea, vomiting, hypercarbia asthma, or chronic obstructive pulmonary disease (COPD).

The device could be further improved during the mass production for the commercial distribution stage, in terms of accuracy, performance, contraindications, disinfecting mechanisms, maintenance guidelines, calibration procedure, materials for mechanical parts \& outer covers, the geometry of the final assembly, nosepiece, etc. Long-term clinical trials are also recommended before the device is introduced into the market.

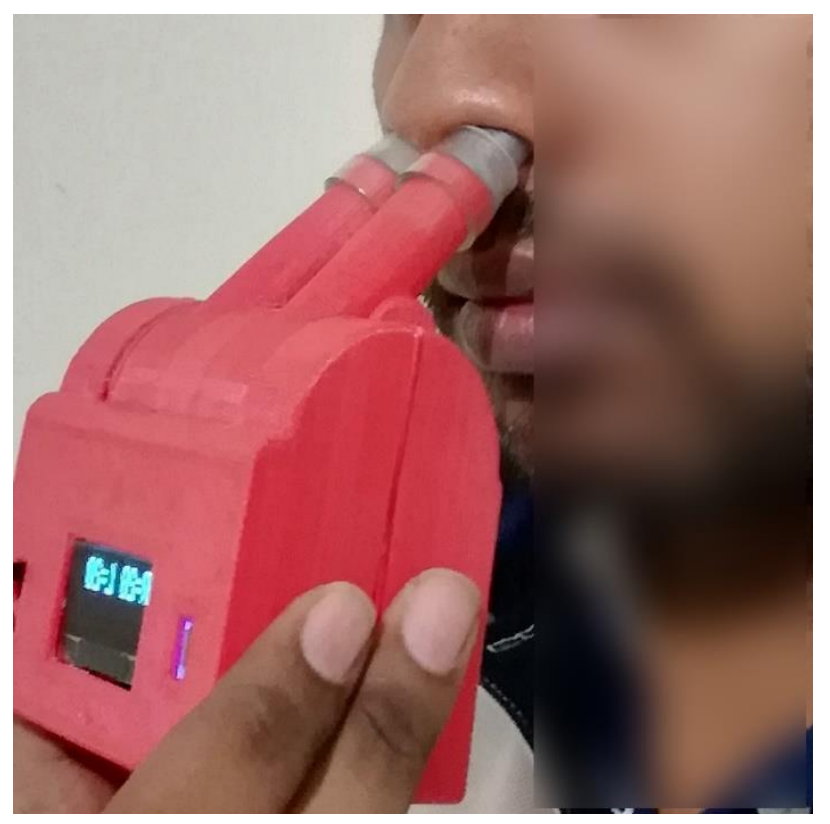

Figure 6. Using the NAMS for measurements 


\section{CONCLUSIONS}

According to the above clarification, it can be concluded that nasal airflow detection and flow rate measuring system has been successfully developed with the main intention to detect and measure the peak nasal exhaled airflow. Nasal airflow rate is very important when a Rhinologist or a physician examines a patient for an initial diagnosis in assessing nasal patency. The developed device is a very unique novel device in many aspects. NAMS is capable of measuring the nasal exhaled peak airflow rate of the two nasal cavities individually and simultaneously and it is a portable device. With this device, the patient needs to inhale from the mouth and exhaled from the nose, as usual breath, and importantly, no extra effort to exhale required compared to other existing mechanisms. It also allows measuring the number of breaths per minute (to monitor breathing style), temperature, and the relative humidity of the airflow of two nasal cavities separately. This can be used as an initial diagnostic tool for Septum Deviation, Nasal Polyps, Nosebleeds, Rhinitis, Nasal Fractions, Nasal Cycles, Breathing Style, etc... as suggested in previous studies [1-3]. Also, Data storing and retrieving facilities are available with the help of the specifically developed application software.

\section{ACKNOWLEDGMENT}

The facilities provided by the Department of Physics, University of Colombo is highly acknowledged.

\section{REFERENCES}

[1] Browning, I.B., D’Alonzo, G.E., Tobin, M.J. (1990). Importance of respiratory rate as an indicator of respiratory dysfunction in patients with cystic fibrosis. Chest, 97(6): 1317-1321. https://doi.org/10.1378/chest.97.6.1317

[2] Yuan, G., Drost, N.A., McIvor, R.A. (2013) Respiratory rate and breathing pattern. McMaster University Medical Journal: Clinical Review, 10(1): 23-25. https://www.mangalam.nl/wpcontent/uploads/2018/12/Breathing-Pattern.pdf.

[3] Chaaban, M., Corey, J.P. (2011). Assessing nasal airflow: Options and utility. Proceedings of the American Thoracic Society, $\quad 8(1)$ : $\quad 70-78$. https://doi.org/10.1513/pats.201005-034rn

[4] Henke, K.G., Dempsey, J.A., Badr, M.S., Kowitz, J.M., Skatrud, J.B. (1991). Effect of sleep-induced increases in upper airway resistance on respiratory muscle activity. Journal of Applied Physiology, 70(1): 158-168. https://doi.org/10.1152/jappl.1991.70.1.158

[5] Eccles, R. (1996). A role for the nasal cycle in respiratory defence. European Respiratory Journal, 9(2): 371-376. https://doi.org/10.1183/09031936.96.09020371

[6] Mitchell, D.M., Gildeh, P., Dimond, A.H., Collins, J.V. (1986). Value of serial peak expiratory flow measurements in assessing treatment response in chronic airflow limitation. Thorax, 41(8): 606-610. https://doi.org/10.1136/thx.41.8.606

[7] Chandra, R.K., Patadia, M.O., Raviv, J. (2009). Diagnosis of nasal airway obstruction. Otolaryngologic Clinics of North America, 42(2): 207-225. https://doi.org/10.1016/j.otc.2009.01.004

[8] Ottaviano, G., Fokkens, W.J. (2016). Measurements of nasal airflow and patency: A critical review with emphasis on the use of peak nasal inspiratory flow in daily practice. Allergy, 71(2): 162-174. https://doi.org/10.1111/all.12778

[9] Bermüller, C., Kirsche, H., Rettinger, G., Riechelmann, H. (2008). Diagnostic accuracy of peak nasal inspiratory flow and rhinomanometry in functional rhinosurgery. The Laryngoscope, 118(4): 605-610. https://doi.org/10.1097/MLG.0b013e318161e56b

[10] Prasun, D., Jura, N., Tomi, H., Pertti, R., Markus, R., Erkki, L. (1999). Nasal airway volumetric measurement using segmented HRCT images and acoustic rhinometry. American Journal of Rhinology, 13(2): 97-104. https://doi.org/10.2500/105065899782106706

[11] Primiano F.P. (2020). Measurements of the respiratory system, In: John G.W., Amit J.N. (eds) Medical Instrumentation: Application and Design, 5th Ed. New York: John Wiley \& Sons. ISBN: 978-1-119-45731-2.

[12] Rosengarten, G., Ahmed, B., Marselli, C. (2003). A novel method to measure respiratory airflow rate based on nasal pressure. IFAC Proceedings Volumes, 36(15): 415-419. https://doi.org/10.1016/s1474-6670(17)335395

[13] Ottaviano, G., Lund, V.J., Nardello, E., Scarpa, B., Mylonakis, I., Frasson, G., Iacono, V., Manzato, E., Marioni, G., Staffieri, A. (2014). Peak nasal inspiratory flow: A useful and handy tool for the diagnosis of nasal obstruction in the elderly. European Archives of OtoRhino-Laryngology, 271(9): 2427-2431. https://doi.org/10.1007/s00405-013-2875-4

[14] Issakhov, A., Zhandaulet, Y., Abylkassymova, A., Issakhov, A. (2021). A numerical simulation of air flow in the human respiratory system for various environmental conditions. Theoretical Biology and Medical Modelling, 18(2): 1-12. https://doi.org/10.1186/s12976-020-00133-8

[15] Li, C., Jiang, J., Dong, H., Zhao, K. (2017). Computational modeling and validation of human nasal airflow under various breathing conditions. Journal of Biomechanics, 64: 59-68. https://doi.org/10.1016/j.jbiomech.2017.08.031

[16] WO/2019/193382, Edirisinghe, C.M., Thilakarathna, T.S. (10-10-2019). Nasal Airflow Detection and Flow Rate Measuring System. WIPO International Application No. PCT/IB2018/052251,

https://patentscope.wipo.int/search/en/detail.jsf?docId= WO2019193382

[17] Pinto, V.L., Sharma, S. (2020). Continuous Positive Airway Pressure. [Updated 2020 Jul 31]. In: StatPearls [Internet]. Treasure Island (FL): StatPearls Publishing; 2020 Jan-. Available from: https:/www.ncbi.nlm.nih.gov/books/NBK482178/

[18] Krishnakumar, S., Kaveri, R., Joshi, A. (2021) Sterilization and Maintenance of Hysteroscopy Instruments. In: Tandulwadkar, S., Pal, B. (eds) Hysteroscopy Simplified by Masters. Springer, Singapore. https://doi.org/10.1007/978-981-15-25056 6

[19] Amodio, E., Kuster, S.P., Garzoni, C., Zinkernagel, A.S., Sax, H., Wolfensberger, A. (2020). Disinfecting noncritical medical equipment-Effectiveness of hydrogen peroxide dry mist as an adjunctive method. American 
Journal of Infection Control, 48(8): 897-902. https://doi.org/10.1016/j.ajic.2020.05.016

[20] Weber, D.J., Rutala, W.A., Anderson, D.J., Chen, L.F., Sickbert-Bennett, E.E., Boyce, J.M. (2016). Effectiveness of ultraviolet devices and hydrogen peroxide systems for terminal room decontamination: focus on clinical trials. American Journal of Infection Control, $44(5)$ : e77-e84 https://doi.org/10.1016/j.ajic.2015.11.015

[21] Rutala, W.A., Weber, D.J. (2016). Disinfection and sterilization in health care facilities: An overview and current issues. Infectious Disease Clinics, 30(3): 609-637. https://doi.org/10.1016/j.idc.2016.04.002

[22] Oth, O., Dauchot, C., Orellana, M., Glineur, R. (2019). How to sterilize $3 \mathrm{D}$ printed objects for surgical use? an evaluation of the volumetric deformation of 3D-printed genioplasty guide in PLA and PETG after sterilization by low-temperature hydrogen peroxide gas plasma. The Open Dentistry Journal, 13(1): 410-417. https://doi.org/10.2174/1874210601913010410

[23] Esslinger, S., Grebhardt, A., Jaeger, J., Kern, F., Killinger, A., Bonten, C., Gadow, R. (2021). Additive manufacturing of $\beta$-Tricalcium phosphate components

via fused deposition of ceramics (FDC). Materials, 14(1): 156. https://doi.org/10.3390/ma14010156

[24] Vanaei, H.R., Shirinbayan, M., Costa, S.F., Duarte, F.M., Covas, J.A., Deligant, M., Khelladi, S., Tcharkhtchi, A. (2021). Experimental study of PLA thermal behavior during fused filament fabrication. Journal of Applied Polymer Science, $\quad 138(4)$ : 49747. https://doi.org/10.1002/app.49747

\section{NOMENCLATURE}

AR

LPM

NAMS

OLED

PC

PEFR

PLA

PNIF

$\mathrm{RH}$

RM

RPM acoustic rhinometry

liters per minute nasal airflow detection \& measuring system organic light-emitting diodes personal computer peak expiratory flow rate Poly-Lactic Acid peak nasal inspiratory flow relative humidity rhinomanometry revolutions per minute 